\section{El papel de la simulación como estrategia educativa en la pandemia de COVID-19}

\author{
The role of simulation as an educational strategy in the COVID-19 pandemic
}

\author{
Dra. Ana Lilia Garduño-López,* Dr. Ricardo Eli Guido-Guerra,* \\ Dr. Víctor Manuel Acosta-Nava, ${ }^{\ddagger}$ Dra. Delia Borunda-Nava, ${ }^{\S}$ \\ Dr. Guillermo Domínguez-Cherit"
}

RESUMEN. El aprendizaje basado en simulación se ha utilizado ampliamente para mejorar la respuesta ante situaciones de crisis. Ha tenido un papel importante en la preparación de los equipos de atención de pacientes con infecciones emergentes como el Ébola, la influenza, el síndrome respiratorio agudo severo (SARS) y el síndrome respiratorio del Medio Oriente (MERS). La reciente pandemia ocasionada por el coronavirus (SARS CoV-2) declarada por la Organización Mundial de la Salud (OMS) en marzo de este año 2020, merece especial atención en estos momentos de crisis internacional, donde la enfermedad ha sobrepasado la respuesta de los sistemas de salud de varios países, por lo que se requiere de la capacitación de los equipos de respuesta para reducir los riesgos. Los objetivos propuestos en esta revisión mediante la práctica deliberada, el escenario clínico y la simulación in situ en el paciente con sospecha o infección por COVID-19 tratan de sistematizar la colocación y retiro del equipo de protección personal (EPP), el manejo de la vía aérea y el abordaje del paciente en paro cardiaco, con la finalidad de mejorar las habilidades técnicas y fortalecer las habilidades no técnicas. Además, durante el desarrollo de estas estrategias, se pueden identificar y abordar situaciones inesperadas, detectar algún compromiso de la seguridad del paciente y/o el personal, lo cual permite rectificar estas deficiencias y optimizar la capacidad de respuesta. Asimismo, permite reflexionar y analizar las emociones del personal para prevenir situaciones adversas en torno al desempeño de los trabajadores de salud. Con esto, se busca fortalecer la seguridad y la calidad en la atención de los pacientes durante el curso de esta pandemia.

ABSTRACT. Simulation-based learning has been widely used to improve response to crisis situations. It has played an important role in preparing care teams for patients with infections such as Ebola, influenza, severe acute respiratory syndrome (SARS) and middle east respiratory syndrome (MERS). The recent pandemic caused by the coronavirus (SARS CoV-2) declared by the World Health Organization (WHO) in March of this year 2020, requires special attention in these moments, where the disease has exceeded the response of the health systems in several countries, so it is necessary training of response teams to reduce risks. The objectives proposed in this review through deliberate practice, the clinical scenario and simulation in situ, in the patient with suspected or infected by COVID-19, try to systematize the placement and removal of personal protective equipment (PPE), the management of the airway and the approach of the patient in cardiac arrest, in order to improve technical skills and strengthen non-technical skills. Furthermore, during the development of these strategies, unexpected situations can be identified and addressed, some compromise in patient and/or staff safety can be detected, which allows these deficiencies to be rectified and response capacity to be optimized. Likewise, it allows to reflect and analyze the emotions of the staff to prevent adverse situations regarding the performance of health workers. With this, it seeks to strengthen the safety and quality of patient care during the course of this pandemic.

\section{Revista Mexicana de \\ Anestesiología}

Octubre-Diciembre 2020

Vol. 43. No. 4. pp 305-314

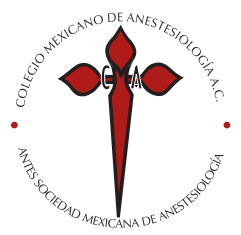

Palabras clave:

Aprendizaje basado en simulación, práctica deliberada, simulación in situ, COVID-19, pandemia.

Keywords:

Simulation-based learning, deliberate practice, on-site simulation, COVID-19, pandemic.

* Médico Adscrito al Departamento de Anestesiología.

‡ Jefe de Departamento de Anestesiología.

$\S$ Coordinadora del Centro de Desarrollo de Destrezas Médicas (CEDDEM).

\| Subdirector de la División de Medicina Crítica.

Instituto Nacional de Ciencias Médicas y Nutrición «Salvador Zubirán» (INCMNSZ).

Solicitud de sobretiros: Dra. Ana Lilia Garduño-López Instituto Nacional de

Ciencias Médicas y Nutrición «Salvador Zubirán».

Vasco de Quiroga Núm. 15, 1er piso, Col. Sección XVI, 14000

Alcaldía Tlalpan, Ciudad de México. Teléfono: (55)54 8709 00,

Ext. 5020 y 5021

E-mail: ana.gardunol@incmnsz.mx

Recibido para publicación: 14-04-2020

Aceptado para publicación: 10-07-2020
L a simulación ha jugado un papel importante en la preparación de equipos médicos ${ }^{(1)}$ para atender a pacientes con infecciones emergentes ${ }^{(2)}$ como el Ébola $^{(3-8)}$, cepas virulentas de influenza ${ }^{(9,10)}$, síndrome respiratorio agudo severo (SARS) $(11,12)$ y el síndrome respiratorio del Medio Oriente ${ }^{(8,13,14)}$. Recientemente, el coronavirus (SARS-CoV2) se ha sumado a este tipo de infecciones, por su reciente emergencia en la ciudad de Wuhan en diciembre del 2019, siendo actualmente una de las enfermedades con mayor diseminación a nivel mundial debido a que no se ha encontrado un tratamiento efectivo contra este virus ${ }^{(15)}$. Hasta el 15 de julio se habían confirmado $13,119,239$ infectados y 573,752 muertes en el mundo, donde Estados Unidos de Norteamérica ocupaba el primer lugar(16). Esta enfermedad fue declarada pandemia 
Tabla 1: Práctica para la colocación y retiro del EPP.

Colocación y retiro del EPP

Objetivo general

Objetivos específicos de aprendizaje

Descripción

Modelaje

Repetición y corrección de la habilidad o destreza

Realimentación

Evaluación
Que el profesional de la salud, realice correctamente la colocación y retiro del equipo de protección personal por medio de práctica deliberada y doble verificación, para mejorar su desempeño durante la pandemia del COVID-19

- Que identifique las diferencias del equipo de protección personal (EPP) necesario para la protección de contagio por gotas vs procedimientos que producen aerolización

- Que realice la colocación adecuada del EPP

- Que realice el retiro adecuado del EPP

Una descripción general de las actividades a realizar durante la práctica:

- Sustento teórico de la infección del coronavirus (pre-briefing)

- Sustento teórico del uso de EPP de acuerdo al grado de exposición: gotas o por aerolización

- Conocer el equipo de protección personal

- Realizar la práctica deliberada de colocación y retiro del EPP

La duración de cada una de las actividades a desarrollar:

- Resumen de aspectos teóricos

- Duración del modelaje

- Repetición del participante

- Corrección de la técnica (doble verificación)

$30 \mathrm{~min}$

$30 \mathrm{~min}$

$30 \mathrm{~min}$ (2 veces colocación y retiro)

$30 \mathrm{~min}$

Se crearán equipos de 2, en cada pareja se asignará el rol de vestimenta a un participante y el otro será un verificador que leerá en voz alta la lista de cotejo y de pasos secuenciales para la colocación y retiro del EPP, se deberán invertir los papeles al término de la actividad

Equipo de protección personal para la práctica por cada 2 participantes:

Pijama quirúrgica hospitalaria

1 par de botas

1 gorro quirúrgico

1 cubrebocas N95

1 par de goggles

1 bata quirúrgica

1 escafandra

1 careta facial

3 pares de guantes

Gel antibacteriano

Caja de guantes extra

Espejo de cuerpo completo

Ayudas cognitivas disponibles

Bolsa de desechos

Posterior al modelaje, el procedimiento será realizado por cada pareja invirtiendo roles las veces que sea necesario hasta que se domine la técnica y sea verificada por el instructor. Si logra el dominio de cada uno de los pasos, deberá continuar hasta que el participante realice el procedimiento en forma óptima y disminuya el tiempo de colocación a no más de 10 minutos y el tiempo de retiro a no más de 20 min.

Se deberá realizar en cada uno de los pasos o secuencias desarrolladas de la técnica de forma oportuna observadas por el instructor y este debe realizar una guía oportuna de mejora

Instrumentos de evaluación: lista de cotejo, ayudas cognitivas para los asistentes

EPP de acuerdo con la recomendación de la OMS y nuestras recomendaciones institucionales

\section{Pasos para la colocación de EPP}

Retirarse todos los objetos personales, pueden dejarse los anteojos y, si es el caso, el pelo debe ser recogido mediante una coleta. Hidratación del personal e ir al servicio sanitario

Colocar botas quirúrgicas 2 pares, 1 impermeable

Realizar higiene de manos con solución alcohólica o colocar alcohol gel en cada uno de los pasos

Colocar primer par de guantes (internos)

Colocarse gorro quirúrgico

Colocarse cubrebocas N95, acomodarla en cara y comprobar que no hay fuga

Colocarse equipo de protección ocular (elegir), visores desechables, goggles reusables y/o careta completa

Colocarse bata quirúrgica impermeable, escafandra (si está disponible), segundo y tercer par de guantes 
Continuación de la Tabla 1: Práctica para la colocación y retiro del EPP.

Retiro de EPP

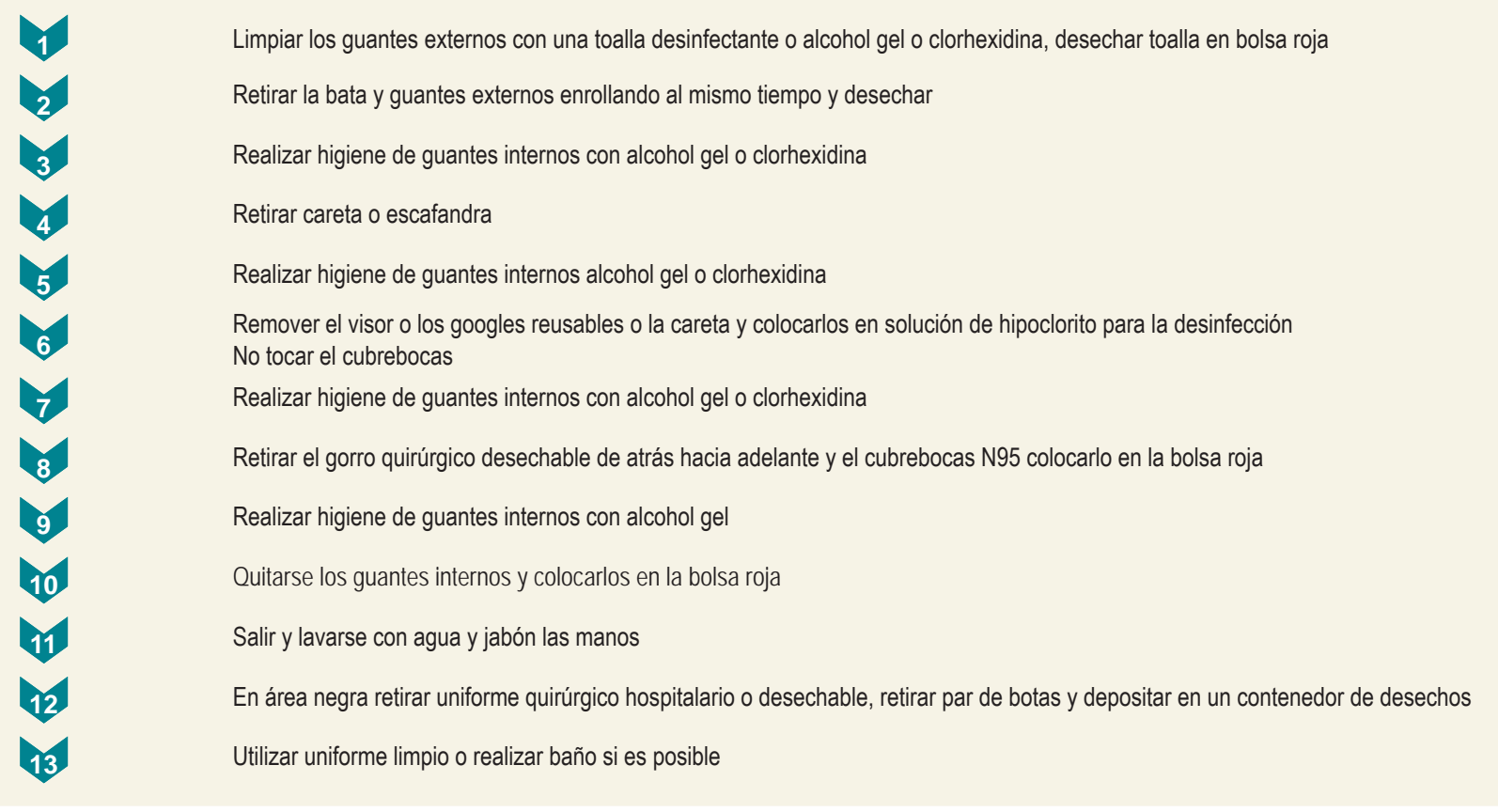

en marzo del 2020 y ha alertado a nuestro sistema de salud en México, donde se han iniciado medidas y estrategias para la mitigación de la enfermedad. Mientras tanto, todo el personal de salud y disciplinas afines debe estar capacitado para brindar atención a los pacientes infectados sin poner en riesgo su seguridad. Actualmente, un estándar de cuidado hospitalario es la protección y el bienestar de los proveedores de atención médica, al tiempo que se mantiene una fuerza laboral suficiente para responder ante las enfermedades, en esta ocasión el compromiso de todo el personal de salud debe incluir conocer a fondo las características fisiopatológicas de la infección ocasionada por SARS-CoV-2, fundamental para la planificación pandémica actual ${ }^{(17)}$.

Esta revisión se dirige a residentes de anestesiología, anestesiólogos y disciplinas afines; con la finalidad de optimizar la respuesta ante la pandemia ocasionada por el COVID-19. El aprendizaje basado en simulación tiene una amplia gama de herramientas que ayudan a la capacitación del personal, a continuación se plantean diversos objetivos como son la práctica deliberada del personal con la colocación y retiro del equipo de protección (EPP), la sistematización segura del manejo de la vía aérea en pacientes infectados y el desarrollo de escenarios clínicos para la toma de decisiones, como la resucitación cardiopulmonar en el paciente con sospecha o COVID positivo, así como la simulación in situ para la planeación estratégica de la ejecución de todos los procedimientos en el medio hospitalario de trabajo y la atención de los pacientes.

Durante el aprendizaje basado en simulación se pueden adquirir tanto habilidades técnicas como no técnicas. Para esta pandemia es importante mejorar ambos tipos de habilidades. Dentro de las habilidades no técnicas encontramos el liderazgo, el trabajo en equipo, la comunicación efectiva, la conciencia situacional y la toma de decisiones ${ }^{(18)}$, los cuales son factores importantes en la atención médica, su optimización puede reducir el error del sistema y fallas en la coordinación de los equipos de atención ${ }^{(19-22)}$. Una reciente revisión sistemática de incidentes críticos en unidades de cuidados intensivos encontró que las fallas en dominios no técnicos contribuyeron a una gran proporción de errores médicos ${ }^{(20)}$, por lo tanto, las mejoras en estas habilidades pueden traer beneficios al sistema de atención.

\section{PRÁCTICA DELIBERADA PARA LA COLOCACIÓN Y EL RETIRO DE EQUIPO DE PROTECCIÓN PERSONAL (EPP)}

En simulación, la adquisición de habilidades técnicas requiere de una práctica deliberada, es decir, una serie planificada de pasos secuenciales con oportunidades para la repetición guiada y monitorizada, con la finalidad de mejorar un dominio en particular ${ }^{(23)}$.

El desarrollo de la experiencia en la colocación y el retiro del equipo de protección personal (EPP) por más fácil que 
Tabla 2: Práctica deliberada para el manejo de la vía aérea.

Manejo de vía aérea en COVID-19

Objetivo general

Objetivos específicos de aprendizaje

Descripción

Modelaje
Que el equipo de manejo de vía aérea ejecute los aspectos técnicos y no técnicos de manejo de la vía aérea en pacientes con COVID-19

- Lograr una comunicación efectiva, realizar trabajo en equipo y favorecer mejoras en el entorno

- Detección por parte del profesional de posibles problemas con el EPP (empañamiento de goggles, acaloramiento, dificultad para habilidades psicomotoras como la intubación)

- Analizar el desempeño de la intubación mediante secuencia rápida con el EPP

- Uso adecuado del filtro antiviral para la reducción de aerosolización

- Minimizar riesgos y manejo adecuado de equipo contaminado

Práctica deliberada in situ en equipo

Requisitos:

- Dominar el proceso de colocación y retiro de equipo de protección personal

- Tener conocimientos sólidos teóricos prácticos sobre manejo de vía aérea fácil y difícil en paciente crítico

Una descripción general de las actividades a realizar durante la práctica:

- Identificación de las variantes particulares en el ambiente de trabajo

- Revisión de las consideraciones teóricas del manejo de la vía aérea en paciente con COVID-19

- Planeación meticulosa del manejo de la vía aérea

- Revisión de comunicación y trabajo en equipo

- Ejecución del procedimiento de manejo de vía aérea

La duración de cada una de las actividades a desarrollar:

- Resumen de aspectos teóricos $30 \mathrm{~min}$

- Duración del modelaje $30 \mathrm{~min}$

- Descanso $5 \mathrm{~min}$

- Repetición de los participantes $\quad 30 \times 2$

- Descanso entre cada repetición $5 \mathrm{~min}$

- Corrección de la técnica (doble verificación) 30 min

Se conforma un grupo de 4 participantes, se asignarán los siguientes roles: líder/asistente de vía aérea (AsisVA), operador de la vía aérea (OpVA), circulante interno (CcInt) y circulante externo (CcExt)

El material necesario depende del abordaje de vía aérea a práctica:

- Equipo de protección personal de acuerdo al rol (Figura 1)

- Videolaringoscopio

- Circuito con filtro antiviral y bolsa mascarilla

- Monitor

- Equipo de aspiración

- Maniquí para manejo de vía aérea

- Tubos endotraqueales de varios calibres

- Mascarilla laríngea

- Guía metálica

- Jeringas rotuladas con fármacos

- Listas de cotejo de material

- Sonda de alimentación

Técnica

1. Circulante externo (CcExt). Da lectura en voz alta y verifica la ejecución de cada uno de los pasos

2. Líder. Establece un plan de manejo individualizado. Hace explícito que se usará mediante instrucciones claras y mantiene circuito cerrado de comunicación

a. Alienta al equipo a hablar si alguien tiene una observación que crea relevante

b. Asigna roles a los miembros del equipo

c. Se excluye personal innecesario para evitar exposición de riesgo

3. Operador vía aérea (OpVa). Verifica que se reúna todo el equipo y material acorde a la estrategia de manejo elegida. Usa lista de cotejo. Se contempla colocación de sonda de alimentación después de la intubación

a. Cuando está listo lo comunica al líder

4. Circulante interno (CcInt). Verifica que el equipo se coloca correctamente el EPP y lo comunica al líder

5. Líder. Comunica al personal encargado que el equipo está listo para recibir al paciente

6. Circulante externo. Disponible para pasar equipo extra al circulante interno

7. Cclnt. Colocado justo en la puerta. Disponible para pasar equipo que se pueda requerir en una emergencia

8. Ingresa el paciente al sitio asignado para realizar la intubación

9. Todos. Se colocan en posición (Figura 1)

10. Asistente vía aérea (AssisVA). Coloca monitorización

11. OpVA. Preoxigenación 5 minutos $\mathrm{FiO}_{2} 100 \%$ con la cabeza del paciente elevada $45^{\circ}$ a. Si el paciente recibe $\mathrm{O}_{2}$ por máscara facial o puntas de alto flujo, descontinúa el flujo de oxígeno para evitar aerosolizar 
Continuación de la Tabla 2: Práctica deliberada para el manejo de la vía aérea.

b. Coloca máscara facial que selle óptimamente, con filtro antiviral y conectado a circuito Bain o bolsa autoinflable

12. Líder. Ejecuta inducción con secuencia rápida y lo comunica al equipo

13. AsisVA. Realiza presión cricoidea en caso de estómago lleno

14. OpVA. Realiza sello óptimo con técnica V-E

15. AsisVA. Apoya ventilar manualmente si es necesario

16. Líder. Monitoriza el tiempo de administración de relajante. Con dosis completa de suxametonio 50 seg; rocuronio 70 seg

17. Indica el momento de la intubación

18. OpVA. Realiza videolaringoscopía e introduce tubo endotraqueal a profundidad óptima

19. Asis. Ayuda con manipulación laríngea externa en caso necesario

20. OpVA. Solicita retiro de guía

21. Asis. Retira guía e infla globo, conecta directamente filtro antiviral, verifica conexiones firmes del circuito. Notifica al equipo que el circuito está conectado y cerrado

22. Líder. Inicia la ventilación mecánica

23. Asis. Fija el tubo. Verifica la expansión torácica y capnografía si se tiene

24. Comunica al equipo el estatus

25. OpVA. Coloca sonda de alimentación

26. Asis. Apoya al OpVA para disponer apropiadamente del material contaminado

27. CcInt. Actúa como monitor en el retiro de EPP

Repetición y corrección de la habilidad o destreza

Retroalimentación

Evaluación
Posterior al modelaje, el procedimiento será realizado en equipo las veces que sea necesario hasta que se domine la técnica y sea verificada por los instructores

Se deberá realizar en cada uno de los pasos o secuencias desarrolladas de la técnica de forma oportuna observadas por los instructores Instrumentos de evaluación: lista de cotejo, ayudas cognitivas para los asistentes parezca, requiere de una compresión tridimensional, es decir, conocimiento de la fisiopatología de la enfermedad (para saber qué equipo utilizar y en qué momento), la familiaridad con los pasos secuenciales para la colocación y el retiro del EPP (para disminuir los riesgos), así como las habilidades psicomotoras con el EPP para realizar otros procedimientos (intubación, colocación de catéter central o arterial, entre otros). Una revisión sistemática evaluó los enfoques para la enseñanza de la realización de procedimientos y demostró la más fuerte evidencia en la estrategia de aprendizaje basado en simulación, la cual es más efectiva en comparación con otros enfoques educativos ${ }^{(24)}$. Un metaanálisis específicamente evaluó la enseñanza de habilidades técnicas en la Unidad de Cuidados Intensivos para médicos y se encontró a la simulación más efectiva que otros métodos de enseñanza en la adquisición de habilidades ${ }^{(22)}$. Un estudio aleatorizado encontró mejoras en la intubación endotraqueal y en el rendimiento de la intubación en un simulador, se demostró que la habilidad se mantuvo cuatro semanas después del entrenamiento de la práctica deliberada ${ }^{(25)}$. La repetición y la familiarización con la técnica de colocación y retiro del EPP provee al profesional un ambiente emocionalmente seguro. Dentro de las lecciones aprendidas en preparación de los brotes de enfermedades infecciosas, estas actividades son valiosas tanto para la comunidad sanitaria en su conjunto como para cada sitio en particular en previsión de minimizar los riesgos.

Es importante mencionar que la secuencia de colocar el equipo de protección puede variar de acuerdo al hospital y a la disponibilidad de éste, lo que no debe variar es la sistematización del personal, en la colocación y el retiro del EPP para reducir el riesgo de contagio.
En la Tabla 1 se describe la guía para la práctica deliberada de la colocación y retiro de EPP.

\section{PRÁCTICA DELIBERADA PARA EL MANEJO DE LA VÍA AÉREA}

La pandemia de COVID-19 pone una carga sustancial a los sistemas de salud; altera los flujos de trabajo habituales, se difieren las cirugías electivas y los anestesiólogos pueden salir del quirófano para incorporarse a equipos de manejo de vía aérea en áreas que les son poco familiares, con personal con el que no trabajan regularmente, aunado a esto, está la variabilidad de recursos en cada centro, la alta demanda y las dificultades que supone trabajar con el equipo de protección personal (EPP) puesto. Enfrentarse a éstos y otros factores modifica el desempeño de los equipos en situaciones críticas como el manejo de vía aérea en un paciente crítico con alto riesgo de transmisión de infecciones. El entrenamiento es clave para tener éxito y preservar la seguridad en esta situación. La práctica deliberada permitirá entrenar una secuencia compleja de pasos que requiere sincronía precisa de todo el equipo. Dado que el manejo de la vía aérea en el contexto de COVID-19 se lleva a cabo en lugares y situaciones diversas y los factores locales (recursos, personal, espacios) cambian en cada centro, esta práctica en equipo debe realizarse in situ de ser posible, con un maniquí útil para la instrumentación de la vía aérea, en cada lugar contemplado, involucrando a miembros del equipo que regularmente atiendan dichas áreas o formen parte del equipo de manejo de vía aérea. (i.e. urgencias, zona de aislamiento habilitada, UTI, etcétera). 
Existen guías que abordan extensamente la valoración y toma de decisiones en vía aérea ${ }^{(26)}$ y son aplicables de manera general al contexto de COVID-19; sin embargo, existen algunos matices y consideraciones a tomar en cuenta. Se destacan los siguientes:

1. Se debe realizar una sesión informativa previa para compartir un modelo mental con todo el equipo antes de la intubación. Esto debe incluir (pero no se limita a) verbalizar la asignación de roles, verificar el equipo, discutir los desafíos anticipados, la estrategia de manejo de la vía aérea, los planes posteriores a la intubación y la colocación de EPP.

2. La protección personal del equipo es prioritaria.

3. Se recomienda minimizar procedimientos que produzcan aerosolización.

4. Los dispositivos para ventilar deben ser conectados directamente a un filtro antiviral.

5. El videolaringoscopio es la técnica ideal inicial en la mayoría de los casos.

6. El personal involucrado debe tener el mayor nivel de experiencia y dominio en manejo de vía aérea.

7. El equipo de vía aérea difícil debe ser considerado y debe estar disponible como son: la mascarilla laríngea en caso de ventilación difícil, el fibroscopio como se muestra en la Figura 1. La intubación con fibra óptica con paciente despierto es un procedimiento de muy alto riesgo de aerosolización, sólo debe ser utilizado cuando está claramente indicado, con máximo nivel de protección del equipo.

8. Se recomienda el uso de listas de verificación y ayudas cognitivas.

9. Se debe preferir el manejo temprano de la vía aérea para evitar intubación de emergencia, en condiciones de gran premura, dado que aumentan el riesgo de contaminación.

10. El entrenamiento repetido y trabajo en equipo son clave para un desempeño seguro y eficaz. En la Tabla 2, se describe la guía para la práctica deliberada para el abordaje de la vía aérea.

\section{ESCENARIO CLÍNICO}

Los escenarios de simulación están diseñados para reproducir situaciones clínicas críticas con diferentes elementos como manejo de emociones, la toma de decisiones, dilemas morales e interacciones complicadas con pacientes, familias o miembros de un equipo multidisciplinario ${ }^{(27)}$. Se requiere de una introducción (briefing) y el establecimiento de un contenedor seguro para la ejecución del escenario clínico donde se establezcan las condiciones de lo que se puede hacer y lo que no, así como los contratos de confidencialidad y de ficción para el desarrollo del caso. Este tipo de

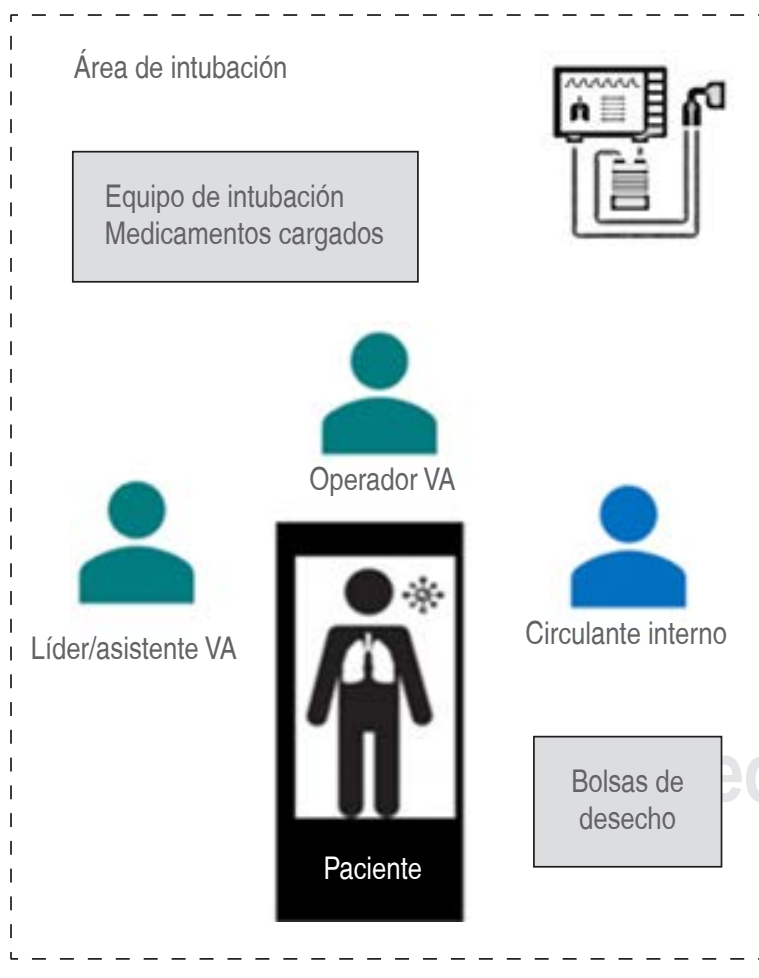

Área externa

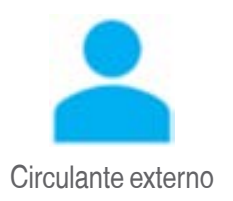

Equipo adicional disponible cerca del área de intubación

- Mascarilla laríngea

- Tubos ET de diferentes

medidas

- Fibroscopio

- Equipo de cricotiroidotomía

- Otros dispositivos para VAD

- Carro de paro cardíaco

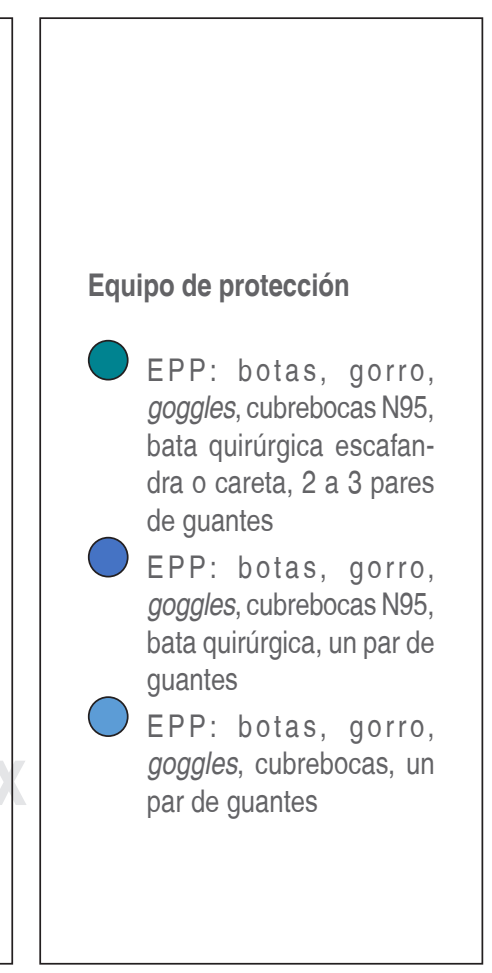

Modificado de: https://www.safeairwaysociety.org/covid19/9(26)

Figura 1: Distribución del equipo para el manejo de vía aérea y EPP de acuerdo con la actividad. 
Tabla 3: Escenario de simulación: paro cardíaco en paciente con sospecha de infección por COVID-19.

Objetivo general:

Conocer las indicaciones y recomendaciones especiales para el manejo de paro cardiaco en paciente con sospecha de infección por COVID-19

\section{Objetivos específicos:}

a) Gestionar el trabajo en equipo, el liderazgo, el establecimiento de roles, la planificación y favorecer la comunicación efectiva

b) Verificar la adecuada colocación del EPP y promover la seguridad del equipo médico, limitar el personal en el área de atención

c) Inicio de compresiones torácicas con las recomendaciones propuestas por la American Heart Association para pacientes con COVID-19 (AHA)

d) Reconocer todas las estrategias ventilatorias durante el paro cardíaco útiles para minimizar riesgo de aerosolización

e) Discutir recomendaciones especiales con el uso de desfibrilador

f) Promover la instrumentación de la vía aérea temprana por expertos

g) Lograr un adecuado manejo del equipo contaminado para disminuir riesgos

h) Iniciar cuidados postparo después del retorno a la circulación: establecer parámetros iniciales de ventilación mecánica

i) Reflexionar sobre las emociones generadas durante el escenario clínico y discutir las indicaciones de atención de paro cardíaco en pacientes con COVID-19

j) Detección por parte del profesional de posibles problemas con el EPP y buscar soluciones (acaloramiento, empañamiento de goggles, dificultad para las compresiones cardíacas, etc.)

k) Verificar el retiro del EPP sin contaminación

l) Utilizar las listas de verificación y guía de «desempeño esperado» de manejo de apoyo cardíaco básico y avanzado en paciente COVID-19 propuestas por la AHA para el apoyo del debriefing https://cpr.heart.org/en/resources/coronavirus-covid19-resourcesfor-cpr-training(28)

Descripción del caso clínico:

Masculino de 48 años de edad, con diabetes, hipertensión y sobrepeso, el cual refería tos y fiebre desde hace una semana. El día de hoy comenzó con dolor torácico, razón por la cual acude a urgencias para recibir atención médica, al momento de su llegada presenta saturación de $70 \%$, se le realiza una gasometría y la relación $\mathrm{PaO}_{2} / \mathrm{FiO}_{2}(\mathrm{PAFI})$ es de 110, por lo que requiere intubación para apoyo ventilatorio.

Al momento de realizar la inducción farmacológica para la intubación, el paciente presenta paro cardiorrespiratorio.

Zona donde se ubica el paciente:

Área de urgencias, con camilla con sábanas y almohada, tripié, monitor de signos vitales (simulador)

Moulage

Maniquí de moderada a alta fidelidad caracterizado como paciente con una máscara reservorio y un cubrebocas encima (ver figura 2).

Tiempo

Briefing 10 min, caso clínico: 10 minutos, debriefing 40 minutos, conclusiones: $10 \mathrm{~min}$

Equipo necesario:

- EPP para el personal, mascarilla reservorio y cubrebocas.

- Maniquí de moderada a alta fidelidad para compresiones cardíacas y para manejo de vía aérea

- Equipo para canalización de línea intravenosa con solución salina

- Equipo de intubación

- Filtro antiviral y ambú

- Desfibrilador

- Jeringas rotuladas con fármacos

- Ayudas cognitivas aprendizaje, basado en simulación para el entrenamiento de los equipos de respuesta durante una pandemia, demandan alta competencia emocional por parte de los participantes ligado, por una parte, a la enfermedad, ya que pueden existir diversos sentimientos encontrados ante el posible riesgo de contagio por el coronavirus y, por otro lado, se pueden encontrar dificultades en la ejecución de la habilidades técnicas secundarias al uso del EPP. En la Tabla 3 se describe un ejemplo de un escenario clínico de un paciente COVID positivo con sus objetivos de aprendizaje y el material requerido. Es necesario que el alumno tenga conocimientos previos de apoyo cardíaco básico y avanzado para la correcta ejecución del escenario.

\section{Seguridad emocional, debriefing y motivación adquirida durante el escenario clínico}

Los instructores de simulación tienen la oportunidad de dirigirse al profesional para el análisis del rendimiento en su conjunto durante el escenario clínico, con la ayuda de un componente esencial de este método de aprendizaje, el debriefing, definido como la conversación entre los participantes, útil para reflexionar y reconocer sus emociones durante el caso, además permite el análisis del desempeño durante éste, así como el papel de los procesos de pensamiento y las habilidades psicomotrices para mejorar o mantener su rendimiento en situaciones futuras ${ }^{(27,29-31)}$.

En este sentido, es muy importante que se genere un ambiente seguro para reflexionar, partiendo de las emociones propias de cada participante, con el objetivo de analizar cómo hacer frente a ellas, de forma interna y externa, para salvaguardar los intereses del participante, con la oportunidad de prevenir un estado de disonancia de identidad, relacionado con el agotamiento físico y mental, así como la insatisfacción profesional durante

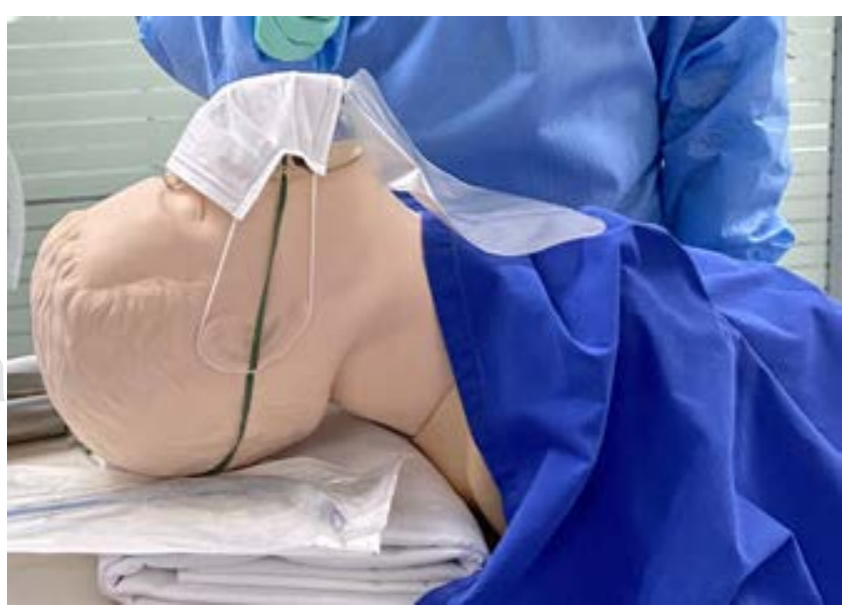

Figura 2: Maniquí con máscara reservorio y cubrebocas. 
la pandemia ${ }^{(29)}$. Además es una oportunidad para reflexionar sobre el impacto de las emociones en la atención al paciente, el trabajo en equipo, la toma de decisiones y sobre el bienestar de los profesionales de la salud. En un estudio reciente, se describió la importancia de lidiar con las emociones durante una sesión informativa después de un encuentro simulado con un paciente, el discutir el impacto de esto en los participantes es relevante para mejorar los resultados ${ }^{(30)}$.
Para que el debriefing sea eficiente y no amenazante, es esencial que el instructor sea capaz de examinar y revelar sus propios modelos mentales con los que interpreta la situación clínica observada. Este tipo de debriefing permite proporcionar dosis específicas de feedback adaptado a las necesidades individuales del participante, y de esta forma ayudarle a modificar sus acciones en situaciones críticas, así como mejorar sus modelos mentales. Es importante discutir

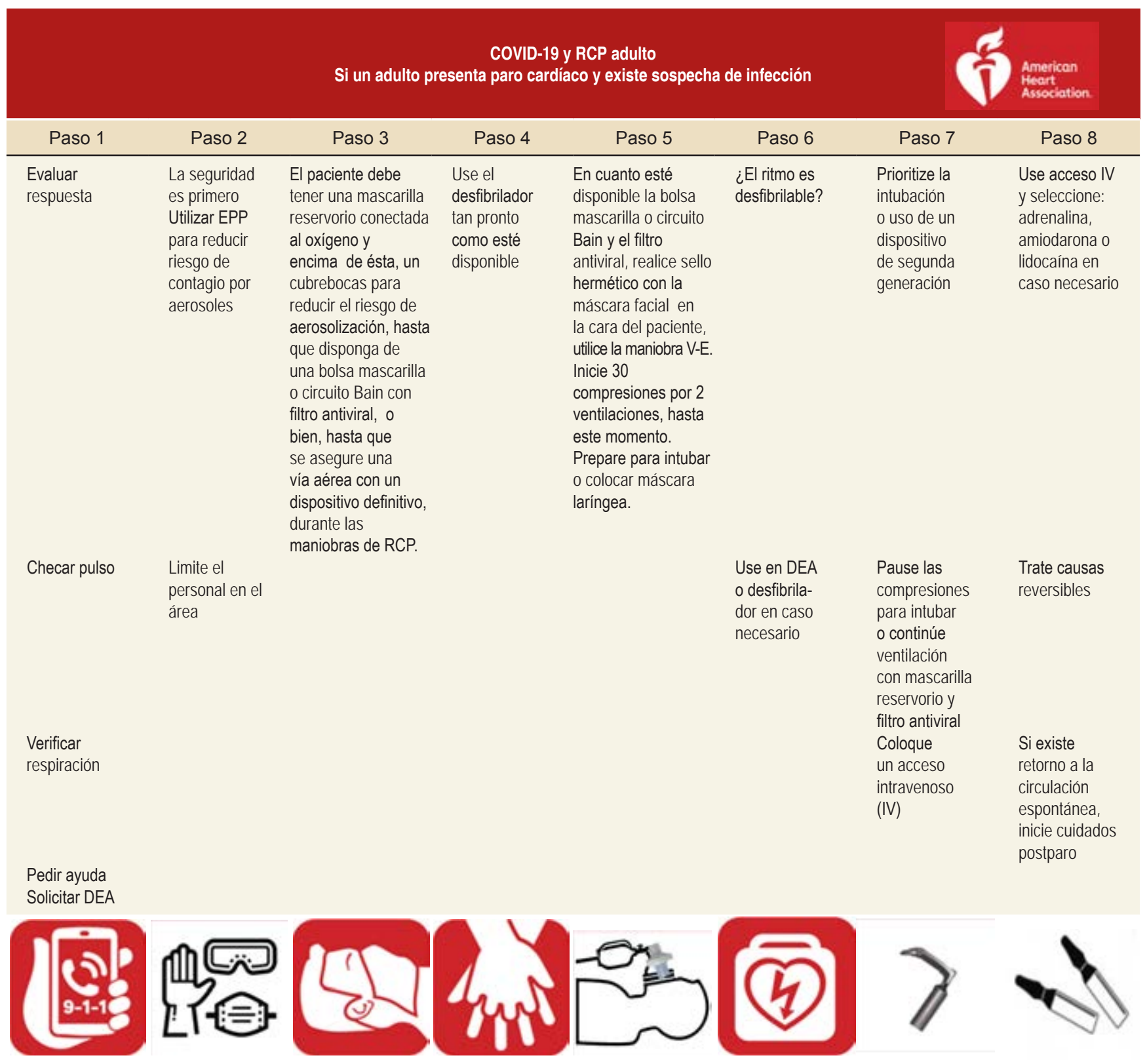

Modificado de: COVID-19 and Adult CPR. Disponible en: https://cpr.heart.org/en/resources/coronavirus-covid19-resources-for-cpr-training(29)

Figura 3: Guía de desempeño esperado en resucitación cardiopulmonar en paciente con COVID-19. 
también los problemas a los que se enfrentaron durante la atención de la crisis, así como el impacto de las destrezas con el EPP completo, con la finalidad de llegar a conclusiones que mejoren la práctica en futuras situaciones de la vida real ${ }^{(31)}$.

Durante el debriefing, el instructor puede utilizar «guías de desempeño esperado» (Figura 3) para la práctica reflexiva, con la finalidad de incorporar aprendizaje nuevo y valioso que aporte recomendaciones específicas para la atención de los pacientes en paro cardíaco durante el brote de COVID-19.

\section{SIMULACIÓN IN SITU}

La simulación in situ permite al personal de una institución formarse simultáneamente en su propio lugar de trabajo y utilizar el equipo médico que utilizan habitualmente, el objetivo de realizarse en el sitio permite la familiaridad del personal con el área. Es necesario el transporte del equipo de simulación y los maniquíes al sitio de trabajo para la planeación en la ejecución de las prácticas. La adaptación de los escenarios a situaciones de emergencia de la vida real permite reflejar durante las simulaciones los objetivos de aprendizaje que se han hecho evidentes durante estas actuaciones. Por ello, los participantes perciben estas simulaciones como extremadamente relevantes en su práctica diaria ${ }^{(15)}$.

La preparación para una pandemia implica considerar las diferentes fases y niveles de la atención. La reconversión de hospitales COVID exige actualmente protocolos que incluyan al aprendizaje basado en simulación para evitar errores en la cadena de atención. En el entorno del quirófano y de las áreas críticas, estas medidas incluyen la modificación de la infraestructura y de los procesos, la gestión del personal y los pacientes, las estrategias de prevención de infecciones y las recomendaciones clínicas. Puede ser un desafío alinear los intereses y las preocupaciones de todas las partes; sin embargo, creemos que estas medidas de contención y simulación in situ son necesarias para optimizar la calidad de la atención brindada a los pacientes con COVID-19 y para reducir el riesgo de transmisión viral a otros pacientes o trabajadores de la salud ${ }^{(15,32)}$.

Es importante detectar las necesidades de cada hospital, para poder plantear los mecanismos de cuidado y prevención durante la pandemia, y aunque, podría pensarse que el aprendizaje basado en simulación es un lujo innecesario en tiempos de crisis, es importante resaltar que son estas situaciones momentos de oportunidad donde se pueden generar beneficios significativos mediante este tipo de aprendizaje. Existen varios retos por venir mientras no exista vacuna o tratamiento para COVID 19, como lo es la nueva normalidad, el retorno a los quirófanos, la educación de médicos residentes, entre otros; por lo tanto, lo que se plantea en esta revisión son sólo ejemplos de cómo mejorar las prácticas de los equipos de atención, durante la contingencia; sin embargo los escenarios y las prácticas pueden variar de acuerdo a los objetivos y las necesidades de cada sitio(33).

\section{CONCLUSIÓN}

El aprendizaje basado en simulación se ha utilizado ampliamente para mejorar la respuesta ante situaciones de crisis $^{(34)}$. Los objetivos propuestos en esta revisión mediante la práctica deliberada, el escenario clínico y la simulación in situ tratan de buscar de manera proactiva amenazas latentes y posibles errores en el control de la diseminación de la infección, especialmente durante momentos de crisis que resultan ser más vulnerables y estresantes como lo es una pandemia. Los ejercicios de simulación permiten identificar y abordar situaciones inesperadas, detectar probable compromiso de la seguridad del paciente y/o el personal, lo cual permite rectificar estas deficiencias, mejorar la capacidad de respuesta y la preparación ante escenarios desafiantes. Asimismo, permite reflexionar y analizar las emociones del personal para prevenir situaciones adversas en torno al desempeño de los trabajadores de salud. Esto finalmente se refleja en tiempos de activación más rápidos, con el objetivo de optimizar la calidad en la atención de los pacientes durante este problema de salud mundial. 


\section{REFERENCIAS}

1. Binstadt ES, Dahms RA, Carlson AJ, Hegarty CB, Nelson JG. When the learner is the expert: a simulation-based curriculum for emergency medicine faculty. West J Emerg Med. 2019;21:141-144.

2. Huang CY, Tsai YS, Wen TH. A network-based simulation architecture for studying epidemic dynamics. Simulation, 2010;86:351-368.

3. Gaba DM, Howard S, Fish KJ, Smith BE, Sowb YA. Simulation-based training in anesthesia crisis resource management (ACRM): a decade of experience. Simulation \& Gaming. 2001;32:175-193.

4. Phrampus PE, O’Donnell JM, Farkas D, Abernethy D, Brownlee K, Dongilli $\mathrm{T}$, et al. Rapid development and deployment of ebola readiness training across an academic health system: the critical role of simulation education, consulting, and systems integration. Simul Healthc. 2016;11:82-88.

5. Adams JJ, Lisco SJ. Ebola: urgent need, rapid response. Simul Healthc. 2016;11:72-74.

6. Abualenain JT, Al-Alawi MM. Simulation-based training in Ebola personal protective equipment for healthcare workers: experience from King Abdulaziz University Hospital in Saudi Arabia. J Infect Public Health. 2018;11:796-800.

7. Rojo E, Oruña C, Sierra D, García G, Del Moral I, Maestre JM. Simulation as a tool to facilitate practice changes in teams taking care of patients under investigation for Ebola virus disease in Spain. Simul Healthc. 2016;11:89-93.

8. Palamidessi-Domínguez J, Valdivia de la Fuente M, Rubio-Muñoz JJ, Alcántara-Carmona S, Palacios-Castañeda D, Martínez-Sanz N, et al. Training on the care of patients with respiratory syndrome of middle east-coronavirus and ebola virus based on clinical simulation. Intensive Care Med Exp. 2015;3:A732.

9. Toner E, Waldhorn $\mathrm{R}$ What hospitals should do to prepare for an influenza pandemic. Biosecur Bioterror. 2006;4:397-402.

10. Brazzi L, Lissoni A, Panigada M, Bottino N, Patroniti N, Pappalardo F, et al. Simulation-based training of extracorporeal membrane oxygenation during H1N1 influenza pandemic: the Italian experience. Simul Healthc. 2012;7:32-34.

11. Khoo KL, Leng PH, Ibrahim IB, Lim TK. The changing face of healthcare worker perceptions on powered air-purifying respirators during the SARS outbreak. Respirology. 2005;10:107-110.

12. Abrahamson SD, Canzian S, Brunet F. Using simulation for training and to change protocol during the outbreak of severe acute respiratory syndrome. Crit Care. 2006;10:R3.

13. Atwa H, Al-Rabia M. Integration of a simulation-based educational model in the medical virology curriculum: a special reference to the recently identified middle east respiratory syndrome-coronavirus (MERS-CoV). MedEdPublish. 2014;3:39.

14. Elcin M, Onan A, Odabasi O, Saylam M, Ilhan H, Daylan Kockaya P, et al. Developing a simulation-based training program for the prehospital professionals and students on the management of middle east respiratory syndrome. Simul Healthc. 2016;11:394-403.

15. Wong J, Goh QY, Tan Z, Lie SA, Tay YC, Ng SY, et al. Preparing for a COVID-19 pandemic: a review of operating room outbreak response measures in a large tertiary hospital in Singapore. Can J Anaesth. 2020. doi: 10.1007/s12630-020-01620-9.

16. Gelb AW, Morriss WW, Johnson W, Merry AF; International Standards for a Safe Practice of Anesthesia Workgroup. World Health OrganizationWorld Federation of Societies of Anaesthesiologists (WHO-WFSA) International Standards for a Safe Practice of Anesthesia. Can J Anaesth. 2018;65:698-708.

17. Poole DN, Escudero DJ, Gostin LO, Leblang D, Talbot EA. Responding to the COVID-19 pandemic in complex humanitarian crises. Int J Equity Health. 2020;19:41.
18. https://www.bbc.com/mundo/noticias-51705060

19. Walsh CM, Scaffidi MA, Khan R, Arora A, Gimpaya N, Lin P, et al. Nontechnical skills curriculum incorporating simulation-based training improves performance in colonoscopy among novice endoscopists: Randomized controlled trial. Dig Endosc. 2020 Jan 7. doi: 10.1111/den.13623.

20. McCallister JW, Gustin JL, Wells-Di Gregorio S, Way DP, Mastronarde JG. Communication skills training curriculum for pulmonary and critical care fellows. Ann Am Thorac Soc. 2015;12:520-525.

21. Curtis JR, Back AL, Ford DW, et al. Effect of communication skills training for residents and nurse practitioners on quality of communication with patients with serious illness: a randomized trial. JAMA. 2013;310:2271-2281.

22. Beal MD, Kinnear J, Anderson CR, Martin TD, Wamboldt R, Hooper L. The effectiveness of medical simulation in teaching medical students critical care medicine: a systematic review and meta-analysis. Simul Healthc. 2017;12:104-116.

23. Ericsson KA. Acquisition and maintenance of medical expertise: a perspective from the expert-performance approach with deliberate practice. Acad Med. 2015;90:1471-1486.

24. Huang G, McSparron J, Balk E. Procedural instruction in invasive bedside procedures: a systematic review and meta-analysis of effective teaching approaches. BMJ Qual Saf. 2016;25:281-294.

25. Mayo PH, Hackney JE, Mueck JT, Ribaudo V, Schneider RF. Achieving house staff competence in emergency airway management: results of a teaching program using a computerized patient simulator. Crit Care Med. 2004;32:2422-2427.

26. Brewster D, Chrimes N, Fraser F, Groombridge C, Higgs A, Humar $\mathrm{M}$, et al. Consensus statement: safe airway society principles of airway management and tracheal intubation specific to the COVID-19 adult patient group. Med J Aust. 2020. Disponible en: https://www. safeairwaysociety.org/covid19/

27. Del Moral I, Maestre JM. A view at the practical application of simulation in professional education. Trends Anaesth Crit Care. 2013;3:146-151.

28. https://cpr.heart.org/-/media/cpr-files/resources/covid-19-resourcesforcpr-training/english/kj1424_covid19_and_cpr_public_200408_ac.pdf? $\mathrm{la}=$ en\&hash=5B7D15B2D817CEC34EC145ADF52763AB98E92F74

29. Carvalho-Filho MA, Schaafsma E, Tio RA. Debriefing as an opportunity to develop emotional competence in health profession students: faculty, be prepared! Sci Med. 2018;28:12.

30. Minehart RD, Rudolph J, Pian-Smith MC, Raemer DB. Improving faculty feedback to resident trainees during a simulated case: a randomized, controlled trial of an educational intervention. Anesthesiology. 2014;120:160-171.

31. Maestre J, Rudolph J. Teorías y estilos de debriefing: el método con buen juicio como herramienta de evaluación formativa en salud. Rev Esp Cardiol. 2015;68:282-285.

32. Fregene T, Nadarajah P, Buckley J, Bigham S, Nangalia V. Use of in situ simulation to evaluate the operational readiness of a highconsequence infectious disease intensive care unit. Anaesthesia. 2020;75:733-738.

33. Dieckmann P, Torgeirsen K, Qvindesland SA, Thomas L, Bushell V, Langli Ersdal $\mathrm{H}$. The use of simulation to prepare and improve responses to infectious disease outbreaks like COVID-19: practical tips and resources from Norway, Denmark, and the UK. Adv Simul (Lond). 2020;5:3.

34. Maestre JM, Szyld D, Del Moral I, Ortiz G, Rudolph JW. The making of expert clinicians: reflective practice. Rev Clin Esp (Barc). 2014;214:216-220. 